\title{
Isotactic and Syndiotactic Pentacontamers (50mers) of Methyl Methacrylate Obtained by Fractionation with Preparative SFC: The GPC Analysis of the 50mers and Their Stereocomplex ${ }^{\dagger}$
}

\author{
Koichi Ute, Nobuo MiYatake, Yasuko Osugi, \\ and Koichi HaTADA* \\ Department of Chemistry, Faculty of Engineering Science, \\ Osaka University, Toyonaka, Osaka 560, Japan
}

(Received March 25, 1993)

\begin{abstract}
Highly isotactic (it-) and highly syndiotactic (st-), 25mers and 50mers of MMA have been isolated from it- and st-PMMAs, respectively, by means of the preparative supercritical fluid chromatography (SFC) using carbon dioxide/ethanol as mobile phase and silica gel as stationary phase. The 25 mers and 50 mers with definite molecular weight (2561 and 5064) were useful as the standard materials in GPC for the calibration of molecular weight and the direct evaluation of instrumental spreading. The $i t-50$ mer eluted faster than the $s t-50 \mathrm{mer}$ in the GPC using THF as eluent. When a solution of a $1: 1$ mixture of the $i t$ - and $s t-50$ mers in THF was subjected to the GPC measurement at $3^{\circ} \mathrm{C}$, the chromatogram showed two peaks at the elution volumes corresponding to the 50 and 100 mers. The elution peak for the 100 mer suggests that the it- and $s t-50$ mers form stereocomplex in a $1: 1$ ratio. The peak area for the stereocomplex varied from 0 to $9.0 \%$ of the whole chromatographic peaks, depending on the conditions (time and temperature) under which the mixed solution of the $i t$ - and $s t-50 \mathrm{mers}$ was allowed to stand. Stereocomplex formation was not observed for a mixture of the $i t$ - and $s t-25$ mers by similar GPC experiments.
\end{abstract}

KEY WORDS Oligomer / Poly(methyl methacrylate) / Supercritical Fluid Chromatography / Gel Permeation Chromatography / Molecular Weight Distribution / Uniform Polymer / Tacticity / Stereospecific Living Polymerization / Stereocomplex /

Various types of oligomers have been prepared as the low molecular weight analogues of synthetic polymers. However, only a limited number of examples have been known as the linear and uniform (monodisperse) oligomers with a degree of polymerization (DP) exceeding 50. Those examples are oligoethylenes $^{1-3}$ and oligo(oxyethylene)s, ${ }^{4}$ both of which were synthesized by the repeated coupling of the homogeneous building-blocks.

Much interest has been devoted in recent years to the chromatographic separation of oligomer mixtures. Particularly, supercritical fluid chromatography (SFC) is one of the most promising techniques for the separation of high DP oligomers, ${ }^{5-8}$ because supercritical mobile phase offers the advantage of high diffusibilities along with widely controllable power of solvation. In the previous paper, ${ }^{9}$ we reported the fractionation of highly isotactic ( $i t$-) and highly syndiotactic (st-) oligo(MMA)s by means of preparative SFC; the individual oligomers from

\footnotetext{
† Stereoregular Oligomers of Methyl Methacrylate VII. Part VI, $c f$. ref 9 .

* To whom correspondence should be addressed.
} 
the 19 mer to the 29 mer obtained in several tens milligrams each were analyzed by ${ }^{1} \mathrm{H}$ and ${ }^{13} \mathrm{C}$ NMR spectroscopies and by differential scanning calorimetry. The series of oligo(MMA) samples served to elucidate the DP dependence of glass transition temperature. Some differences in solid-state properties were also shown to exist between the PMMAs with and without molecular weight distribution (MWD).

In order to extend the studies on the uniform oligo(MMA)s, we have attempted to isolate the $i t$ - and st-50mers whose molecular weight is 5064 (including terminal groups). The $i t$ - and $s t$-oligo(MMA)s with this extent of molecular weight are expected to form stereocomplex ${ }^{10,11}$ when they are mixed under appropriate conditions. Katime and Quintana carried out gel permeation chromatography (GPC) of the mixtures of $i t$ - and st-PMMAs with MWDs $\left(\bar{M}_{w}>1.8 \times 10^{5}\right)$, and examined the complexation stoichiometry in dilute solution. The resulting stereocomplex was highly polydisperse and a part of the mixture gave a microgel which was retained in the filter before the GPC column. ${ }^{12}$ The stereocomplex between the it- and st-50mers avoids such experimental difficulties arising from MWD, and provides a straightforward approach to the complexation studies.

The uniform oligomers with definite molecular weight are interesting not only as the model compounds of polymers but also as reference materials in analytical science. For example, usefulness of pure oligo(ethylene glycol)s ${ }^{4}$ and some dendritic macromolecules $^{13,14}$ as standard materials in GPC was recently stated. We have applied the uniform oligo(MMA)s to the calibration of molecular weight in GPC analysis and also to the evaluation of instrumental spreading in the chromatography.

\section{EXPERIMENTAL}

The $i t$ - and $s t$-PMMAs were prepared as reported previously. ${ }^{15,16}$ Living isotactic polymerization of MMA was initiated with $t$ $\mathrm{C}_{4} \mathrm{H}_{9} \mathrm{MgBr} \quad\left([\mathrm{MMA}] /\left[t-\mathrm{C}_{4} \mathrm{H}_{9} \mathrm{MgBr}\right]=45\right.$ $\mathrm{mol} / \mathrm{mol}$ ) in toluene at $-78^{\circ} \mathrm{C}$ and the reaction was terminated $96 \mathrm{~h}$ after the initiation by adding a small amount of phenol dissolved in toluene to the polymerization mixture. ${ }^{15,17}$ The termination with phenol is necessary for the selective formation of meso diad at the chain-end. ${ }^{17}$ The mixture was poured into methanol and the soluble part (yield $82.7 \%$, $\overrightarrow{D P}=40.8, \bar{M}_{w} / \bar{M}_{n}=1.12, m m: m r: r r=95.9$ : $3.5: 0.6)$ was subjected to fractionation by SFC. Living syndiotactic polymerization reaction was carried out with $t-\mathrm{C}_{4} \mathrm{H}_{9} \mathrm{Li} /(n$ $\left.\mathrm{C}_{4} \mathrm{H}_{9}\right)_{3} \mathrm{Al}([\mathrm{Al}] /[\mathrm{Li}]=3 / 1 \mathrm{~mol} / \mathrm{mol},[\mathrm{MMA}] /$ $\left.\left[t-\mathrm{C}_{4} \mathrm{H}_{9} \mathrm{Li}\right]=40 \mathrm{~mol} / \mathrm{mol}\right)$ in toluene at $-93^{\circ} \mathrm{C}$, and the reaction was terminated with $t$-butanol $48 \mathrm{~h}$ after the initiation. ${ }^{16,17}$ The termination with $t$-butanol gives high syndiotacticity at the chain-end diad. ${ }^{16}$ The polymerization mixture was poured into hexane and the insoluble part (yield 97.7\%, $\overline{D P}=41.4$, $\left.\bar{M}_{w} / \bar{M}_{n}=1.05, m m: m r: r r=0: 7.2: 92.8\right)$ was subjected to SFC.

SFC was performed on a JASCO SUPER200 chromatograph equipped with a HewlettPackard 5890 column oven. A $10 \mathrm{~mm}$ i.d. $\times 250 \mathrm{~mm}$ column packed with non-bonded silica gel (Develosil 100-5, Nomura Chemical Co., Ltd., particle size $5 \mu \mathrm{m}$ ) was used. The system consists of two pumps, one is for the delivery of liquefied $\mathrm{CO}_{2}$ as mobile phase (flow rate $\left.=9.0 \mathrm{ml} \mathrm{min}^{-1}\right)$, and the other for ethanol as entrainer (flow rate $=2.5 \mathrm{ml} \mathrm{min}^{-1}$ ). Concentration of the entrainer in the mobile phase was kept constant throughout the experiments. The fluid pressure was controlled by a backpressure regulator to $205 \pm 1 \mathrm{~kg} \mathrm{~cm}^{-2}$. The column temperature was set initially to $80^{\circ} \mathrm{C}$ for the it-PMMA or $95{ }^{\circ} \mathrm{C}$ for the st-PMMA, and the temperature was lowered at a rate of $1^{\circ} \mathrm{Cmin}^{-1}$ to $40^{\circ} \mathrm{C}$ immediately after the injection of sample solution to the column. Chromatograms were recorded using a UV detector operated at a wave length of $220 \mathrm{~nm}$. 


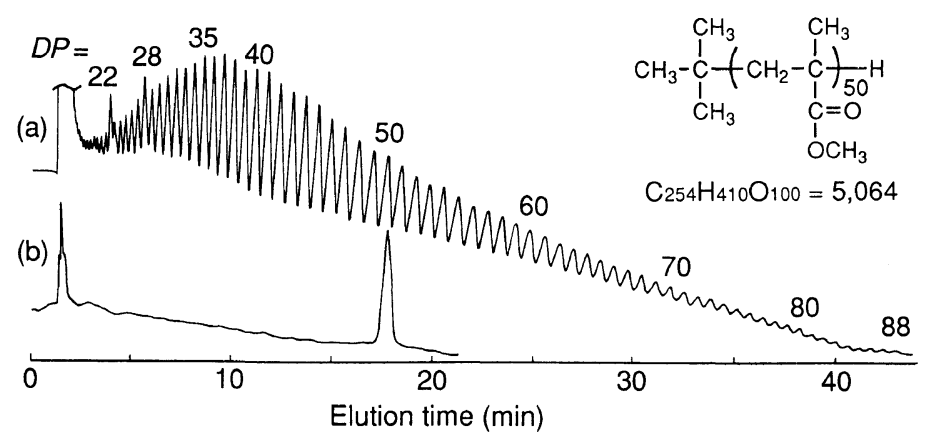

Figure 1. SFC traces of isotactic PMMA $\left(\overline{D P}=40.8, \bar{M}_{w} / \bar{M}_{n}=1.12\right.$, containing the authentic samples of the 22 and 28mers) (a) and the 50mer isolated from (a) (b).

GPC was performed on a TOSOH CCPD chromatograph equipped with a Shodex GPC column KF-803 $(8.0 \mathrm{~mm}$ i.d. $\times 300 \mathrm{~mm}$, maximum porosity $=7 \times 10^{4}$ ) employing tetrahydrofuran (THF) as eluent. The flow rate was $0.5 \mathrm{ml} \mathrm{min}^{-1}$. The column temperature was controlled to 25.0 or $3.0^{\circ} \mathrm{C}$ by means of a water-jacket. A Shodex SE-61 RI detector was used. Sample concentrations were $0.10 \%(\mathrm{w} / \mathrm{v})$ for PMMA and $0.05 \%(\mathrm{w} / \mathrm{v})$ for polystyrene standards. The volume of sample solution was 3 to $30 \mu \mathrm{l}$. The polystyrene standards $\left(\bar{M}_{n}=\right.$ 42800, $\bar{M}_{\mathrm{w}} / \bar{M}_{n}=1.01 ; \bar{M}_{n}=17000, \bar{M}_{w} / \bar{M}_{n}=$ $1.04 ; \quad \bar{M}_{n}=6200, \quad \bar{M}_{w} / \bar{M}_{n}=1.04 ; \quad \bar{M}_{n}=2800$, $\left.\bar{M}_{w} / \bar{M}_{n}=1.05\right)$ were obtained from Toyo Soda Manufacturing Co., Ltd. and Chemco Scientific Co., Ltd.

\section{RESULTS AND DISCUSSION}

\section{Fractionation of PMMA by Means of SFC}

Figure 1a shows an SFC curve of the itPMMA $\left(\overline{D P}=40.8, \bar{M}_{w} / \bar{M}_{n}=1.12\right)$ prepared by the stereospecific living polymerization of MMA initiated with $t-\mathrm{C}_{4} \mathrm{H}_{9} \mathrm{MgBr}$ in toluene. The polymer was separated into the individual homologues from the $16 \mathrm{mer}$ to the $88 \mathrm{mer}$. The chromatographic conditions were adjusted to achieve the best separation for the components from the 40 mer to the 60 mer. A small amount of the authentic samples of it-22mer and it-28mer, which were isolated in our previous work, ${ }^{9}$ was added to the it-PMMA as the internal standards of $D P$; the elution peaks due to the $22 \mathrm{mer}$ and the $28 \mathrm{mer}$ are distinguishable by their enhanced intensities. The $D P$ of the authentic samples was based on the results of ${ }^{1} \mathrm{H}$ NMR and FD mass spectroscopies.

Fractionation of the individual components from the $40 \mathrm{mer}$ to the $60 \mathrm{mer}$ was carried out 45 times. The amount of the sample introduced to the column was $25 \mathrm{mg}(50 \mu \mathrm{l}$ of $50 \%(\mathrm{w} / \mathrm{v})$ acetone solution) for each chromatographic run which took about $45 \mathrm{~min}$. The fraction of the it-50mer was purified by repeated fractiona tion by the SFC since the fraction contained a small amount of lower DP components. The purified it-50mer gave a single peak in the chromatogram recorded under the identical conditions (Figure 1b), indicating that the it-50mer was uniform with respect to $D P$. The amount of the uniform it-50mer isolated was $10.6 \mathrm{mg}$.

An SFC curve of the st-PMMA $(\overline{D P}=41.4$, $\left.\bar{M}_{w} / \bar{M}_{n}=1.05\right)$ prepared by the stereospecific living polymerization of MMA initiated with $t-\mathrm{C}_{4} \mathrm{H}_{9} \mathrm{Li} /\left(n-\mathrm{C}_{4} \mathrm{H}_{9}\right)_{3} \mathrm{Al}$ complex in toluene is shown in Figure $2 \mathrm{a}$. The oligomer components from the $23 \mathrm{mer}$ to the $70 \mathrm{mer}$ were resolved completely. The chromatographic conditions were the same as those for the separation of the it-PMMA except that the initial column temperature was set to $95^{\circ} \mathrm{C}$. A small amount of the authentic sample of $s t-24 \mathrm{mer}^{9}$ was added to the $s t$-PMMA as an internal standard. Each fraction from the $30 \mathrm{mer}$ to the $50 \mathrm{mer}$ was collected 47 times. The crude fraction of the 


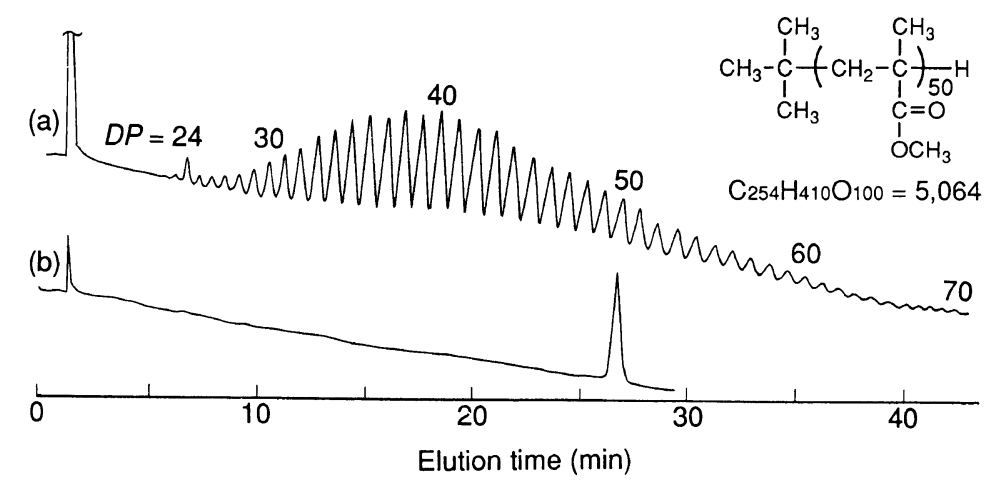

Figure 2. SFC traces of syndiotactic PMMA $\left(\overline{D P}=41.4, \bar{M}_{w} / \bar{M}_{n}=1.05\right.$, containing the authentic sample of the 24mer) (a) and the 50 mer isolated from (a) (b).
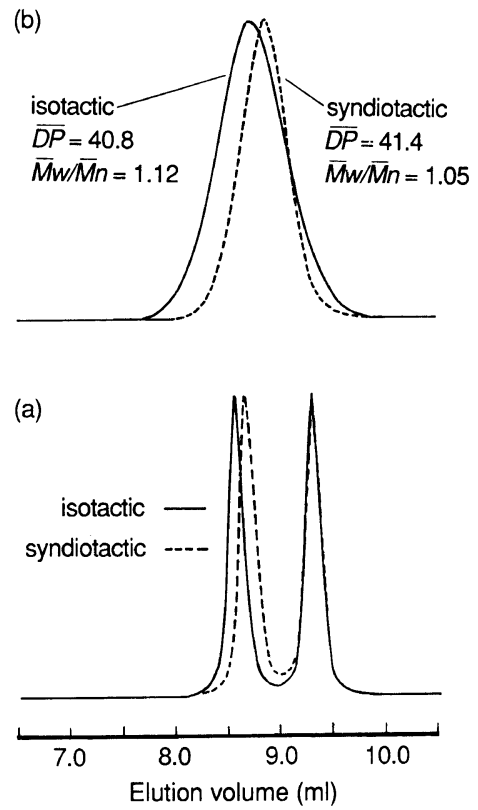

Figure 3. GPC curves of the $1: 1$ mixtures of the isotactic 25 and $50 \mathrm{mers}(-)$ and of the syndiotactic 25 and 50 mers (---) (a), and PMMAs with molecular weight distribution (b).

st-50mer was purified by repeated fractionation. Reinjection of the $s t-50$ mer thus obtained $(16.6 \mathrm{mg}$ ) proved its purity (Figure $2 b$ ).

Use of the Uniform Oligo(MMA)s as the Standard Materials in GPC

The mixtures of $i t-25 \mathrm{mer}^{9}$ and $i t-50 \mathrm{mer}$, and of $s t-25 \mathrm{mer}^{9}$ and $s t-50 \mathrm{mer}$ were prepared and
Table I. Elution volumes ${ }^{\mathrm{a}}(\mathrm{ml})$ of isotactic and syndiotactic uniform oligo (MMA)s in GPC

\begin{tabular}{ccc}
\hline & Isotactic & Syndiotactic \\
\hline $25 \mathrm{mer}$ & $9.325 \pm 0.007$ & $9.364 \pm 0.006$ \\
$50 \mathrm{mer}$ & $8.611 \pm 0.006$ & $8.729 \pm 0.007$ \\
\hline
\end{tabular}

a The average of 5 determinations. Estimated standard deviations are also given.

were subjected to GPC analysis (Figure 3a). The sharpness of the elution curves compares quite favorably with those of the original PMMA samples (Figure $3 \mathrm{~b}$ ). Table I gives the elution volumes at the elution maximum of the uniform oligo(MMA)s. The elution volume was measured five times for each sample, and the average values had standard deviations less than $0.007 \mathrm{ml}$. It was found from the data in Table I that $i t-25$ mer eluted significantly faster than $s t-25$ mer. More distinct difference of elution volumes was observed between the 50mers. These results indicate that the hydrodynamic volume of it-PMMA in THF is larger than that of st-PMMA. A similar phenomenon was found in the GPC measurements of the it-PMMA $\left(\bar{M}_{n}=6.34 \times 10^{4}\right.$, $\left.\bar{M}_{w} / \bar{M}_{n}=1.21\right)$ and st-PMMA $\left(\bar{M}_{n}=6.47 \times\right.$ $\left.10^{4}, \bar{M}_{w} / \bar{M}_{n}=1.13\right)$ carried out in chloroform at $39^{\circ} \mathrm{C} .{ }^{18}$ On the basis of dilute solution 
properties, it has been known that the st-PMMA coil is less extended than the isotactic form in the unperturbed state $\left(\Theta\right.$-solvents) and in several good solvents. ${ }^{19}$

The difference of elution volume due to stereoregularity brings about a problem in the measurement of molecular weight by GPC. When the $\bar{M}_{n}$ of the $i t$-PMMA was determined by employing it-25mer and it-50mer as molecular weight standards, the value agreed well with the $\bar{M}_{n}$ based on the end-group assay by ${ }^{1} \mathrm{H}$ NMR (Table II). However, use of $s t$ $25 \mathrm{mer}$ and $s t-50 \mathrm{mer}$ as standard materials made the $\bar{M}_{n}$ value $8.6 \%$ larger. Similarly, the $\bar{M}_{n}$ of the $s t$-PMMA calculated from the chromatogram calibrated with it-25mer and it-50mer deviated $8.9 \%$ from the $\bar{M}_{n}$ value obtained from the chromatogram calibrated with $s t-25 \mathrm{mer}$ and $s t-50 \mathrm{mer}$. The $\bar{M}_{n}$ values of the PMMAs as determined by the calibration curve with polystyrene standards were in the intermediate.

Although the uniform oligo(MMA) samples are free from MWD (i.e., $\bar{M}_{w}=\bar{M}_{n}$ ), GPC measurements of the 25 mers and 50 mers gave the apparent $\bar{M}_{w} / \bar{M}_{n}$ values ranging from 1.009 to 1.013. The apparent MWD of the uniform oligomers comes from the instrumental spreading of the elution band, and the values show the lower limit of $\bar{M}_{w} / \bar{M}_{n}$ in this GPC instrument. When an ideal chromatographic curve is assumed to be Gaussian, the solute concentration $(C)$ can be expressed by the equation:

$$
C=C_{\max } \exp \left(-x^{2} / 2 \sigma^{2}\right)
$$

where $x$ is the difference between the volume eluted and the volume corresponding to the maximum concentration $\left(C_{\max }\right)$, and $\sigma$ is the standard deviation. ${ }^{20}$ The band width at 0.606 $C_{\max }$ equals to $2 \sigma$. Use of the uniform oligomers permitted us to evaluate the $\sigma$ directly from the chromatogram. The GPC curves of the uniform oligomers fitted well to Gaussian curves and the $\sigma$ was $0.070 \mathrm{ml}$ when the volume of the sample solution was $0.003 \mathrm{ml}$.
Table II. The $\bar{M}_{n}$ and $\bar{M}_{w} / \bar{M}_{n}$ values of isotactic and syndiotactic PMMA samples determined by the GPC calibrated with isotactic and syndiotactic uniform oligo (MMA)s (25 and 50mers)

\begin{tabular}{lccccc}
\hline \multirow{2}{*}{$\begin{array}{c}\text { Reference } \\
\text { material }\end{array}$} & \multicolumn{2}{c}{$i t$-PMMA } & & \multicolumn{2}{c}{$s t$-PMMA } \\
\cline { 2 - 3 } \cline { 5 - 6 } & $\bar{M}_{n}$ & $\bar{M}_{w} / \bar{M}_{n}$ & & $\bar{M}_{n}$ & $\bar{M}_{w} / \bar{M}_{n}$ \\
\hline$i t-25 / i t-50$ & 4111 & 1.131 & & 3853 & 1.054 \\
$s t-25 / s t-50$ & 4466 & 1.171 & & 4229 & 1.062 \\
PSt-mix $^{\mathbf{a}}$ & 4340 & 1.122 & & 4079 & 1.051 \\
$\left({ }^{1} \mathrm{H} \mathrm{NMR}\right)^{\mathrm{b}}$ & 4138 & - & & 4190 & - \\
\hline
\end{tabular}

a A mixture of four polystyrene samples.

${ }^{b}$ Determined from ${ }^{1} \mathrm{H}$ NMR spectra.

\section{Stereocomplex Formation of the Uniform Oligo $(M M A) s$}

It has been known that it- and st-PMMA chains associate to form stereocomplex in certain solvents including THF. ${ }^{21}$ GPC measurements of the complex in THF are usually difficult because the complex gels in the eluent. However, the mixture of the it- and $s t-50$ mers in THF did not produce any gel nor precipitate during the experiments described below. The mixture was prepared by mixing $150 \mu \mathrm{l}$ of each solution in THF $(0.10 \%(\mathrm{w} / \mathrm{v}))$ at room temperature. The column temperature was kept at $3^{\circ} \mathrm{C}$.

Figure 4a shows the GPC curve of the mixture recorded immediately after the preparation of the mixture. The elution peak exhibited a small splitting due to the difference of elution volume between it- and $s t$-50mers as mentioned before. An extra peak appeared at the elution volume corresponding to $c a$. the 100mer $\left(\bar{M}_{n}=1.09 \times 10^{4}\right.$, based on a polystyrene standard) when the chromatogram was measured after the mixture was allowed to stand at $-15^{\circ} \mathrm{C}$ for $5 \mathrm{~h}$ (Figure $4 \mathrm{~b}$ ). The extra peak is attributable to the stereocomplex formed between the it-and st-50mers, since no such peak was detected in the control experi ments carried out for the individual 50mers. 


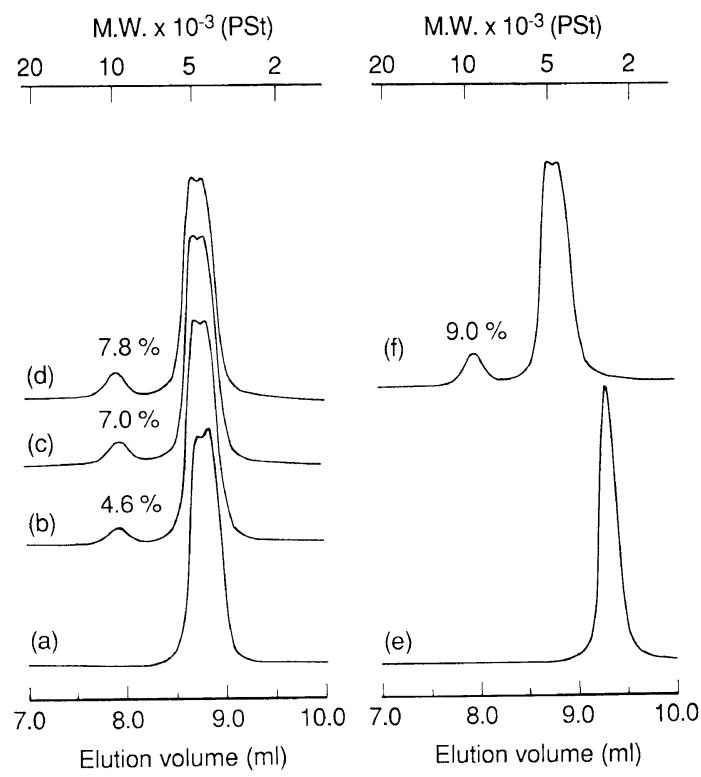

Figure 4. GPC curves of the $1: 1$ mixture of the isotactic and syndiotactic 50 mers stored at $-15^{\circ} \mathrm{C}$ for (a), 0 , (b) 5, (c) 10 , and (d) $32 \mathrm{~h}$. The mixture (d) was allowed to stand at $-30^{\circ} \mathrm{C}$ for additional $24 \mathrm{~h}$ (f). The GPC curve of the mixture of the isotactic and syndiotactic 25 mers stored at $-30^{\circ} \mathrm{C}$ for $96 \mathrm{~h}$ is shown in (e). Eluent, THF.

The monodispersity of the extra peak $\left(\bar{M}_{w} /\right.$ $\left.\bar{M}_{n}=1.007\right)$ indicates that the complex has an exact stoichiometry; it is suggested from the elution volume of the complex that the complexation stoichiometry is it-50mer:st$50 \mathrm{mer}=1: 1$. The stoichiometry of $1: 1 \mathrm{may}$ be supported by the fact that the intensity ratios of the elution peaks due to the remaining $i t$ - and $s t$-50mers (elution volumes of 8.69 and $8.81 \mathrm{ml}$ ) were essentially identical between before and after the appearance of the extra peak. The area of the extra peak was $4.6 \%$ to the whole chromatographic peaks. The area increased to 7.0 and $7.8 \%$ when the chromatography was performed after the mixture was stored at $-15^{\circ} \mathrm{C}$ for 10 and $32 \mathrm{~h}$, respectively (Figure $4 \mathrm{c}$ and $4 \mathrm{~d})$. Keeping this mixture $\left(-15^{\circ} \mathrm{C}, 32 \mathrm{~h}\right)$ for additional $24 \mathrm{~h}$ at $-30^{\circ} \mathrm{C}$ enhanced the area to $9.0 \%$ (Figure $4 \mathrm{f}$ ). In the case where the mixture had been stored at $3^{\circ} \mathrm{C}$ since its preparation, however, the peak due to the stereocomplex did not exceed $5.7 \%$ in its area even after $96 \mathrm{~h}$ (the figure is not shown). Interestingly, a $1: 1$ mixture of $i t$ - and st-25mers did not show such extra peak in the chromatogram even after the mixture was allowed to stand at $-30^{\circ} \mathrm{C}$ for $96 \mathrm{~h}$ (Figure $4 \mathrm{e}$ ).

A number of contradictory results have been published about the stoichiometry of the stereocomplex of PMMA (cf. ref 11). Most authors find the stoichiometry it: $s t=1: 2^{10,21}$ but papers indicating the stoichiometry it : $s t=$ $1: 1^{22}$ or $i t: s t=1: 1.5^{12,23}$ also exist. Chiang et al. ${ }^{24}$ conclude that no defined stoichiometry of the stereocomplex exists, while other authors $^{25}$ assume that the composition lies between it:st $1: 1.5$ to $1: 2$. Although the reasons for the disagreements are not clear, our results on the uniform 50mers suggest the ratio $i t: s t=1: 1$; it should be noted that the complexation stoichiometry discussed in the present work deals with the number of associated molecules whereas the stoichiometries reported in the past concern the ratio of associated monomeric units (or the weight fractions of the two components). Our conclusion, however, is based on the assumption that the hydrodynamic volume of the complex do not differ very much from those of the individual it- and st-components (at least, in a $D P$ range $\leq 100$ ). In order to obtain further information of the composition, the fraction of the extra peak in Figure $4 \mathrm{f}$ was collected several times and was subjected to $500 \mathrm{MHz}{ }^{1} \mathrm{H}$ NMR analysis. Although both it- and st-50mers were detected in the spectrum, determination of the composition was not possible because of a low signal-to-noise ratio of the spectrum (over 30000 scans) and severe overlap of the signals due to a small amount of impurities. Extensive studies are under way using a larger amount of the oligomer samples.

The reason why only a part of the 50mers (maximum 9.0\%) formed the complex may be attributed to imperfect stereoregularity of the 50 mers used. The stereoregularity of the it$50 \mathrm{mer}$ is $m=0.979$ in diad and that of the $s t$ - 
50mer is $r=0.964$. The configurational statistics for the 50 mer chains can be regarded as Bernoullian because the persistence ratios $(=2[m][r] /[m r])$ for the 50 mers are close to unity $(1.17$ for the $i t-50$ mer and 0.96 for the $s t$-50mer). Therefore, the relative abundances of the perfectly $i t$ - and perfectly $s t-50$ mers are calculated to be $m^{49}=0.353$ and $r^{49}=0.166$, respectively. If we assume that 50 consecutive $m$ or $r$ sequences are required for the complexation, the yield of the stereocomplex between the it- and st-50mers used in the present work should be not more than $16.6 \%$. Since the uniform 25 mers did not form stereocomplex, it is suggested that the minimum sequence length necessary for the complexation exists somewhere between 25 and 50.

Spěváček and Schneider reported, from ${ }^{1} \mathrm{H}$ NMR studies on mixtures of it-PMMA $(\overline{D P}=270, m m=97 \%)$ and $s t$-PMMA $(26 \leq$ $\overline{D P} \leq 1700)$ in benzene- $d_{6}$, that the fraction of associated monomeric units in the mixtures increased from $30 \%$ to $89 \%$ as the $r$-triad in the $s t$-PMMA increased from $65 \%$ to $88.5 \%$. On the basis of the results, they concluded that the minimum length of associated syndiotactic sequences required for stereocomplex formation was $c a .10$ monomeric units in aromatic solvents and $c a .3$ such units in the more strongly complexing solvents. ${ }^{23}$ Because THF used in our study is classified as a strongly complexing solvent, our result that the minimum sequence length lies between 25 and 50 is considerably large as compared with their values. The discrepancy is partly due to the fact that the it-PMMA used in their work had a much larger average $D P$ than the uniform oligo(MMA)s employed in the present work. It is suggested that the uniform oligo(MMA)s, which do not have a sufficiently large $D P$ to form three dimensional network (gel) by stereocomplex formation, need longer tactic sequences for the complexation.

Acknowledgments. A part of this work was supported by a Grant-in-Aid for Scientific Research (No. 03453118) from the Ministry of Education, Science, and Culture of Japan.

\section{REFERENCES}

1. I. Bidd and M. C. Whiting, J. Chem. Soc., Chem. Commun., 543 (1985).

2. G. Ungar, J. Stejny, A. Keller, I. Bidd, and M. C. Whiting, Science, 229, 386 (1985).

3. K. S. Lee and G. Wegner, Makromol. Chem., Rapid Commun., 6, 203 (1985).

4. S. Kinugasa, A. Takatsu, H. Nakanishi, H. Nakahara, and S. Hattori, Macromolecules, 25, 4848 (1992).

5. R. E. Jentoft and T. H. Gouw, J. Polym. Sci., B7, 811 (1969).

6. E. Klesper and W. Hartmann, Eur. Polym. J., 14, 77 (1978).

7. F. P. Schmitz and E. Klesper, J. Supercritical Fluids, 3, 29 (1990).

8. K. Hatada, K. Ute, T. Nishimura, M. Kashiyama, T. Saito, and M. Takeuchi, Polym. Bull., 23, 157 (1990).

9. K. Ute, N. Miyatake, T. Asada, and K. Hatada, Polym. Bull., 28, 561 (1992).

10. A. M. Liquori, G. Anzuino, V. M. Corio, M. D'Alagni, P. de Santis, and A. Savino, Nature, 206, 358 (1965).

11. J. Spěváček and B. Schneider, Adv. Colloid Interface Sci., 27, 81 (1987).

12. I. A. Katime and J. R. Quintana, Makromol. Chem., 189, 1373 (1988).

13. C. J. Hawker and J. M. J. Fréchet, J. Am. Chem. Soc., 112, 7638 (1990).

14. T. M. Miller, T. X. Neeman, R. Zayas, and H. E. Bair, J. Am. Chem. Soc., 114, 1018 (1992).

15. K. Hatada, K. Ute, K. Tanaka, Y. Okamoto, and T. Kitayama, Polym. J., 18, 1037 (1986).

16. T. Kitayama, T. Shinozaki, T. Sakamoto, M. Yamamoto, and K. Hatada, Makromol. Chem., Suppl., 15, 167 (1989).

17. K. Ute, T. Asada, N. Miyatake, and K. Hatada, Makromol. Chem., Macromol. Symp., 67, 147 (1993).

18. K. Hatada and T. Kitayama, "Stereoregular Macromonomers with Respect to Main Chain and/or Side Chain" in "Macromonomers, Macroinitiators, Macroiniferters, Macroinifers-Design and Application," M. K. Mishra, Ed., Polymer Frontiers Corp., 1993, p xxx.

19. J. M. G. Cowie, "Solutions of Stereoregular Polymers" in "Light Scattering from Polymer Solutions," M. B. Huglin, Ed., Academic Press, London, 1972, p 605.

20. E. Heftmann, "Chromatography," Van Nostrand 
Reinhold Co., New York, 1975, p 334.

21. E. J. Vorenkamp, F. Bosschen, and G. Challa, Polymer, 20, 59 (1979).

22. T. Miyamoto and H. Inagaki, Polym. J., 1, 46 (1970).

23. J. Spěváček and B. Schneider, Makromol. Chem., 175,
2939 (1974).

24. R. Chiang, J. J. Burke, J. O. Threlkeld, and T. A. Orofino, J. Phys. Chem., 70, 3591 (1966).

25. J. Biros, Z. Masa, and J. Pouchly, Eur. Polym. J., 10, 629 (1974). 